\title{
sciendo
}

\author{
BULGARIAN ACADEMY OF SCIENCES
}

CYBERNETICS AND INFORMATION TECHNOLOGIES • Volume 19, No 2

Sofia 2019 Print ISSN: 1311-9702; Online ISSN: 1314-4081

DOI: $10.2478 /$ cait-2019-0013

\section{D Face Factorisation for Face Recognition Using Pattern Recognition Algorithms}

\author{
Hawraa H. Abbas, Bilal Z. Ahmed, Ahmed Kamil Abbas \\ Department of Electrical and Electronic Engineering, University of Kerbala, Iraq \\ E-mails:Hawraa.h@uokerbala.edu.iq bilal.zu@uokerbala.edu.iq ahmed.kamil@uokerbala.edu.iq
}

\begin{abstract}
The face is the preferable biometrics for person recognition or identification applications because person identifying by face is a human connate habit. In contrast to $2 D$ face recognition, $3 D$ face recognition is practically robust to illumination variance, facial cosmetics, and face pose changes. Traditional 3D face recognition methods describe shape variation across the whole face using holistic features. In spite of that, taking into account facial regions, which are unchanged within expressions, can acquire high performance $3 D$ face recognition system. In this research, the recognition analysis is based on defining a set of coherent parts. Those parts can be considered as latent factors in the face shape space. Non-negative matrix Factorisation technique is used to segment the 3D faces to coherent regions. The best recognition performance is achieved when the vertices of 20 face regions are utilised as a feature vector for recognition task. The region-based $3 D$ face recognition approach provides a $96.4 \%$ recognition rate in FRGCv2 dataset.
\end{abstract}

Keywords: Face recognition, clustering, FRGCv2 dataset, non-negative matrix factorisation.

\section{Introduction}

Biometric traits are physical or behavioral human characteristics that can be classified in pre-defined categories established by humans with the aim of differentiating individuals $[1,2]$. The face is one of the most revealing parts of the human body. Besides being our way of communicating emotions and intentions, it also contains information about the identity of a person, like sex, age, ancestry, weight, etc. The study of the human face is one of strong interest in the shape modeling community under the heading of morphometric. Morphometric studies comprise the quantitative analysis of human head size and face shape. To some degree, certain variations in our face appearance are local. For example, the shape of our eyes is independent of the shape of our lips. Even for a feature as simple as the lips, there are many different subtle variations, such as their width, curvedness, length, etc. therefore, within the context of face recognition, a further distinction can be made into holistic and part- 
based approaches. The effect of part-based face perception in humans has revealed in studies such as the Thatcher illusion [3]. In this study, a portrait photo has edited by placing the eyes and mouth upside down, with respect to the face. Although the inversion of the parts can be readily observed, the shapeless effect it has on the facial appearance as a whole. In machine recognition, a part-based representation can be advantageous, because traits within part are highly correlated with each other, but relatively independent of traits in other part [4]. Therefore, in 3D face recognition, it can provide robustness to facial expression by excluding affected parts [5], or by including only parts of interest [6]. In reconstruction and animation, the expressiveness of a face can be improved by modeling individual parts [7]. Consequently, in this research we study the relation between the face modules (segments) and face recognition. Non-negative Matrix Factorisation (NMF) is used to segment the face to different number of modules, and then the effect of these modules in face recognition accuracy is investigated. The main contribution of this research is using NMF for $3 \mathrm{D}$ face segmentation for face recognition purpose.

The rest of this paper is organised as follows: A brief review for related research is introduced in Section 2. Section 3 presents the proposed method for 3D face recognition. The experimental results are recorded in Section 4. The discussion for the acquired results is introduced in Section 5. Finally, Section 6 concludes the achievements of the proposed method, and it comprise suggestions for future research.

\section{Related work}

Holistic feature-based approaches work directly on the overall triangle mesh or pointcloud of face scans. This kind of methods extract the facial feature or perform the similarity comparison associated to the whole face. One of classical holistic feature relays on using Principal Component Analysis (PCA) and Linear Discriminant Analysis (LDA) for analyzing this face space, PCA and LDA can be applied in different representation pattern with basis vectors to represent high-dimensional space. The range images are projected to the principle component vectors, then these vector coefficients consider as the feature of the range image [8,9]. Another classical holistic method by using Iterative Closest Point (ICP). The optimal parameters of ICP are the ones, which are used to minimize the distance between the query surface and the corresponding reference surface $[9,10]$. For more holistic 3D face recognition methods investigate the survey in [11].

Local region feature based methods are proposed to manage local shape distortion caused by facial expression changes. The principle of these methods is to divide the facial surface into several sub-regions, then carry out the feature extraction, and apply the feature matching process on these separated regions. For example, Amor, Ardabilian and Chen [12] presented an improved region-based approach for 3D face authentication and recognition. They did a manual segmentation on the full-face scan to divide the face surface. Then, they analyze the facial expression depending on anatomical view and empirical experiments, eventually they manually classify the muscles of the face according to the degree of distortion they 
cause. The experiments conducted on ECL-3D-face database including 50 full 3D faces, the best reported recognition rate is $97.86 \%$. In addition, Spreeuwers [13] proposed a fast 3D face recognition method. To deal with the facial expression variance, the author designs a series of 30 overlapping regions over the facial range image. The vectorization form of each region constructs the feature vector, and their dimensionality is reduced by PCA followed by LDA. Then, these region features are being fed into a likelihood classification. The best recognition rate was reported as $95.9 \%$ on FRGCv2 dataset. These methods did the face segmentation either manually or by using the nose tip as basic region and extracted different regions size around it (for more details see also [14, 15]).

NMF has recently attracted much attention and has been shown to be useful in a variety of applications, such as chemometrics [16], pattern recognition [17] and multimedia data analysis [18]. Therefore, in this research, an automatic decomposition approach of faces into small parts using non-negative matrix Factorisation is proposed and the effect of these Factorisation on the face recognition is explored. NMF was used for segmenting the 2D image in [19], while in this paper it is used for the $3 \mathrm{D}$ faces.

\section{The proposed approach}

An overview of the proposed face recognition approach is provided in Fig. 1, and the algorithm's different components are explained below.

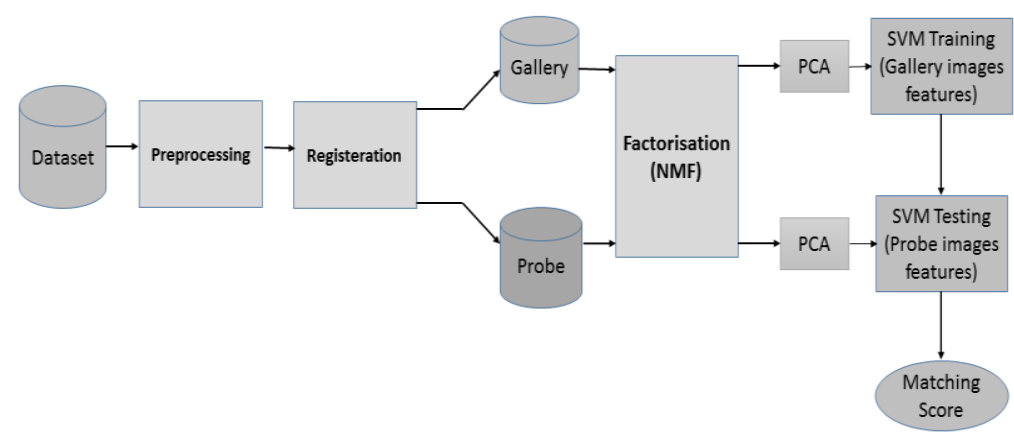

Fig. 1. Block-diagram of the proposed face recognition system

\subsection{Dataset}

The data used in experimental work come from the FRGCv2 dataset. This data set contains 3D facial images of 466 persons, with 22 images per subject, the total number of images are 4007. The Minolta Vivid 910 camera is used to register these images [20]. In this dataset, 2469 3D face images are classified as neutral expression, while the rest of the 4007 images have non-neutral expressions (e.g., disgust, happiness, surprise). In this work, neutral images are used, $80 \%$ randomly used for training and the rest for testing. A total of 1975 images were used as a gallery (training images) and 494 images were used for probing (testing images). 
Pre-processing. Preprocessing operations are aimed to improve the extraction of object information in the given images. The data from 3D scanners frequently suffer from imperfections. These imperfections are generally in the form of noise or holes. Therefore, data preprocessing is a vital and non-trivial step. All scans in FRGCv2 database are preprocessed by using 3DFaceModelsPreprocessing Tool ${ }_{1}$ [21], to eliminate noise and remove some undesired parts such as clothes, neck, ears, and hair from the scanned images. The denoising images were smoothed by using the medium filter, which removes the noise spikes. The holes are detected by searching vertices that have less than eight adjacent vertices, and filled by fitting square surfaces.

\subsection{Registration}

The aims of the registration process are alignment of all faces and find mesh dense correspondence between them. The technical details and algorithms that are used for dense surface model generation are provided in [22]. The following steps give an informal summary for the registration process:

- A random face is taken from the database as an initial template or base mesh.

- Five landmarks annotated manually on the 3D facial image (medial canthus of the right eye, medial canthus of the left eye, nasal tip, right labial commissure, left labial commissure).

- Following the manual landmarks placing on all 3D face surfaces, the mean landmarks for the set is calculated using the Generalized Procrustes Algorithm (GPA) [23].

- Each surface is warped with the mean landmarks using the Thin-Plate Spline (TPS) technique [24], to bring the corresponding landmarks on each face into accurate alignment.

- A k-Nearest Neighbour Rule [25] is used to assign dense mesh correspondences (closest point) with the vertices of a base mesh.

\subsection{Non-negative matrix factorisations}

The massive interest in NMF is the newly discovered ability of NMF to solve challenging clustering problems [26]. NMF has been shown to be a useful tool for analysing multivariate data. In machine learning, the approximation of a matrix by two factorizing low-rank matrices has many clear benefits. Among these benefits are discovering a structure in data, as well as reducing dimensionality and making a way for better generalization. In particular, the non-negativity constraint induces sparsity, causing selection of the variables into groups. In the field of facial analysis, it has shown to combine spatially nearby vertices into coherent parts [27]. Formally, the non-negative Factorisation of a matrix $V \approx W H$, of size $(n \times m)=(n \times k)(k \times m)$, can only be exact if $k \geq \operatorname{rank}(V)$. In practice, $k$ is usually chosen much smaller, and so the Factorisation approximates the original data with a residual matrix that can contain both positive and negative elements.

Assume each column of $V$ is an image of $m$ vertices. Then, by using the approximation $V \approx W H$, one may say that the columns of $W$ correspond to a basis image. Each column of $H$, on the other hand, corresponds to the encoding of each 
image in terms of the basis images. Non-negativity constraints on $V, W$, and $H$ implicitly make for more interpretable and parts-based representation of the data. $W$, $H_{0}$ implies that each element $V_{i j}=w_{i} h_{j}$, is a weighted addition of positive vectors. By limiting both the basis and the encoding to non-negative values, NMF forces the Factorisation to an additive weighted structure (encoding), of non-negative building blocks (bases) [28].

\subsection{Principal Component Analysis (PCA)}

The sub regions feature vector is extracted and dimensionally reduced using PCA. In general, PCA aims to search for the best vector for the distribution of images, and to use this vector to define the subspace of range images. All face images in training group are projected into the principle components space to find out a set of coefficients, which describes the contribution of each face vector in the face space. For identifying a testing face image, it is projected to the trained face space to achieve the corresponding set of coefficients. The vectors of PCA are known as eigenvectors, which correspond to the largest eigenvalues. Meanwhile, the eigenvectors are also known as eigenfaces. Usually, the input images represented as $n$-dimension feature vectors are reduced to a feature vector of $d$-dimensional, because the eigenvectors corresponding to smaller eigenvalues are mostly noise and they usually are neglected [29].

\subsection{Machine learning}

The Support Vector Machine (SVM) is a supervised learning approach that is used in pattern recognition. SVM maps an input sample to high dimensional feature space and works on finding the optimal hyperplane that can minimise the classification error for the training data using the non-linear transformation function. The classifier tries to find the optimal one by maximizing the margin. This margin, as shown in Fig. 2 is the minimal distance between the hyperplane and the training data [30].

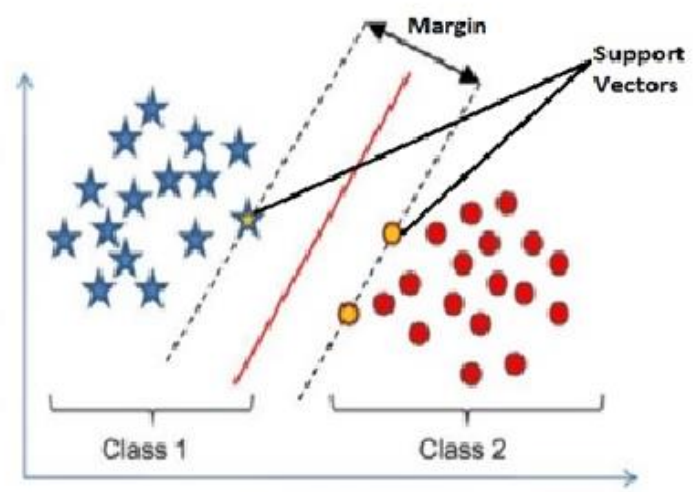

Fig. 2. Margin, supports vectors and hyperplane for SVM

Cross-validation method ( $\mathrm{N}$-fold) is used for evaluating the recognition performance. In this research, the dataset is divided into five subsets. Each time, one of the five subsets are used as the test set and the rest sets are used for training, after that the average error across all five trials is calculated [31]. 


\section{Results}

Although the segmentation method is unsupervised in the sense that there is no example segmentation to learn from, the choice of the number of parts, $K$, is a controlling parameter. In fact, the number of parts is the main controlling parameter for the segmentation of the face. The number of parts defines a scale ranging from 1 (whole face) up to intermediate values of parts to the total number of vertices. Progression along this scale means a reduction for information per part. This reduction is an offset against an increase of information found in the relations between parts. Fig. 3 visualises the full segmentation based on 10 parts, while Fig. 4 visualizes the segmentation based on 20 parts. As the number of parts increases, the size of the features decreases. A range of $K$ value is tested, increasing $K$ value over 20 produce very small parts that might be better combined with other parts. In fact, for the segmentation in more than 35 regions, the face is divided in small contiguous regions that separate different anatomical aspects, but the results are less intuitive to interpret since the regions are small. For a smaller number of parts, remarkable result in the 2-region division is that mouth and nose are combined into one region. According to this result, some relation exists between these anatomical structures. As they coincide in the same region, there must be higher covariance between them, and less interaction with the parts in the other region as shown in Fig. 5. The vertices of 10 parts, 20 parts and 35 parts are used as features for face recognition purpose. Table 1 shows the recognition rate and error rate for 3D face recognition using SVM, while Fig. 6 illustrates the relation between the accuracy and the number of regions.
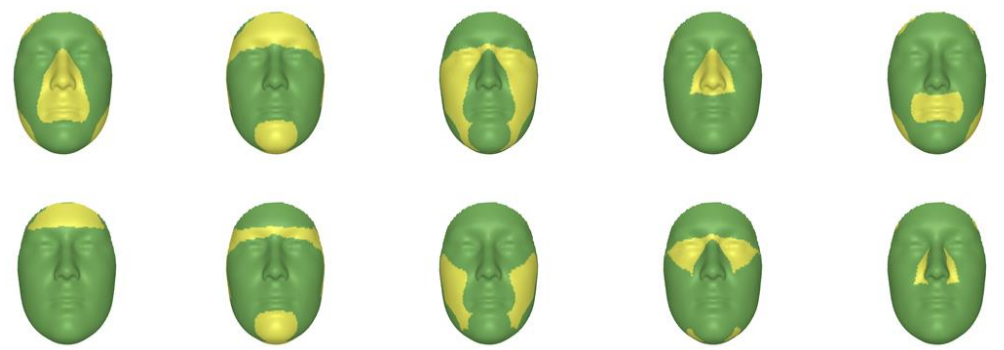

Fig. 3. Visualization of 10 parts
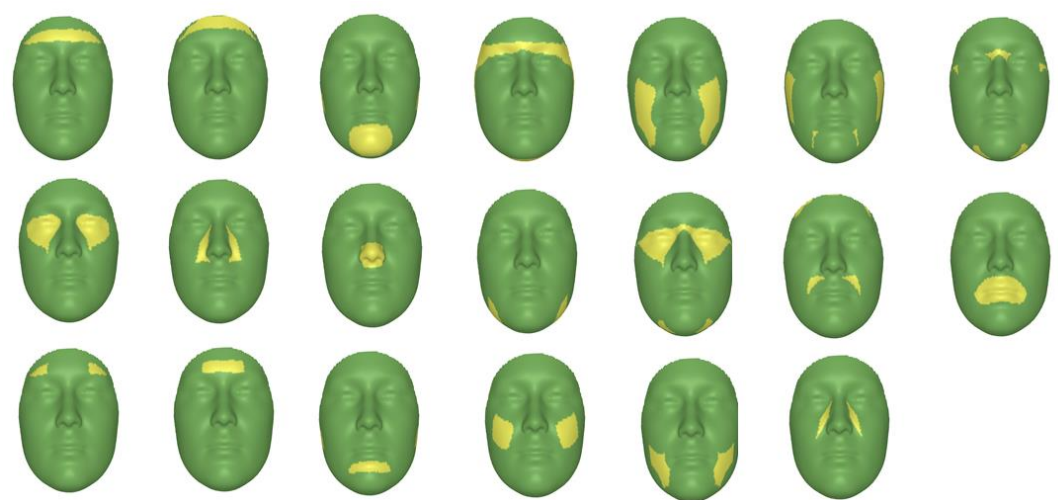

Fig. 4. Visualization of 20 parts 


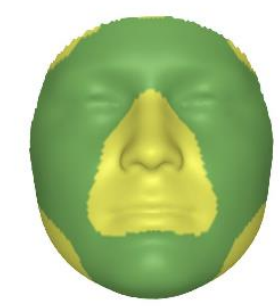

Fig. 5. Visualization of two parts

Table 1. The resultants of Recognition rate and Error rate of 3D face recognition system

\begin{tabular}{|c|c|c|}
\hline Number of parts & Recognition rate & Error rate \\
\hline 10 parts & $92.7 \%$ & $5.2 \%$ \\
\hline 25 parts & $96.4 \%$ & $2.7 \%$ \\
\hline 35 parts & $95.7 \%$ & $5.5 \%$ \\
\hline
\end{tabular}

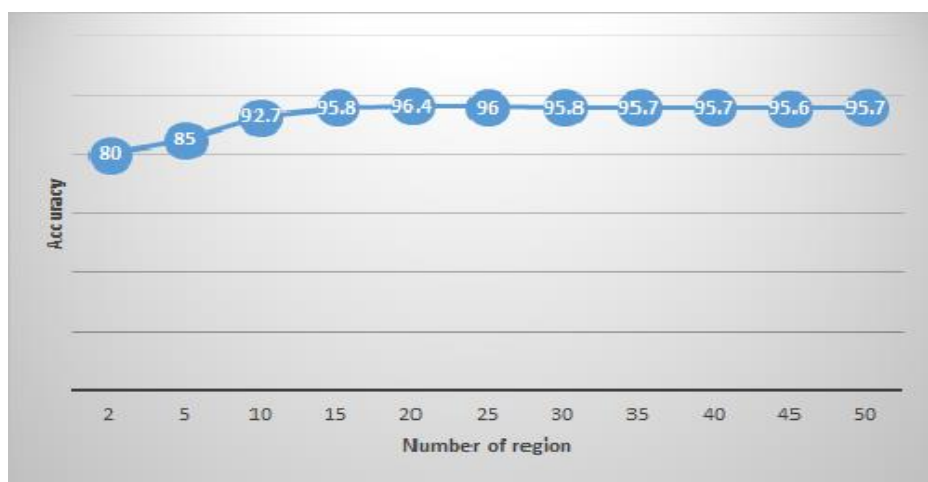

Fig. 6. The recognition accuracies for different number of face regions

\section{Discussion}

Non-negative matrix Factorisation is suited for decomposition of the whole face. NMF results in sparser basis vectors that group strongly correlating vertices. The strongest correlation is found among local groups of vertices. Consequently, the basis vectors obtained can be used to define a statistical decomposition of the face into parts. Face Factorisation is unsupervised segmentation process, the selection of the number of segments is an essential parameter for any segmentation algorithm to avoid under-segmentation and over-segmentation problems. Fig. 7 illustrates several examples of these problems. Under-segmentation, in which the segments appear to describe more than one part of the face. Over-segmentation when segments appear to describe only part of the part. Therefore, an open question remains: what is the correct number of parts that is sufficient for face recognition. Logically, more parts mean smaller parts and vice versa, but further experiments are needed to find a proper criterion for the evaluation. A general trend that can be seen from the results in Fig. 6 is the increase in accuracy when the number of regions is increased, but this is followed by a drop-in performance due to over fitting. The increasing performance 
when segmenting the face in more regions, suggests that investigating the face from a region perspective enhances the recognition rate. Experimental tuning for the $k$ value yield $96.4 \%$ recognition rate when $k=20$.
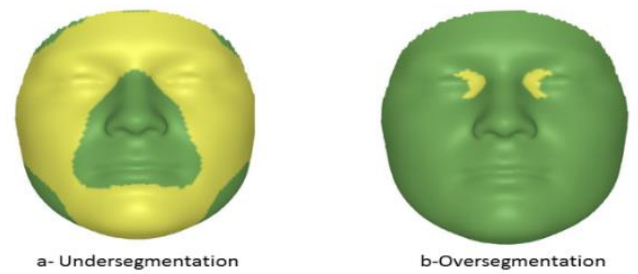

Fig. 7. Under segmentation and over segmentation

A number of studies have investigated face recognition performance using face parts conducted on FRGCv2 dataset. $\mathrm{C} h$ a n g, B o w y e $\mathrm{r}$ and F 1 y n $\mathrm{n}$ [14] divide the 3D face into regions; the center of these regions is the nose. These regions are used for comparison instead of the whole frontal face region. While, $\mathrm{Z} \mathrm{h}$ on g, S u n and T an [15] divided the frontal face into two regions, the upper and the lower face region. They used K-means clustering technique for face segmentation and nearest neighbor classifier for the classification. For the same purpose, L e i, B e n n m o u n and E 1-S a 11 a m [32] collected features from the eyes, forehead and nose regions, these features are represented as a histogram. They used fusion of feature level for evaluation. Table 2 compare the recognition performance of the proposed approach with respect to the performance obtained in other states of art 3D face recognition systems. We can notice the high performance of 3D face recognition system based on face Factorisation. The major novelty in this paper is the automatic face segmentation or partitioning while the segmentation process in the state of art research was done manually.

Table 2. Comparison with other methods

\begin{tabular}{|c|c|c|c|}
\hline Reference & Recognition rate & Number of regions & Dataset \\
\hline $\begin{array}{c}\text { Faltemier, B ow yer } \\
\text { and F1yn n [33] }\end{array}$ & $93.2 \%$ & 28 & FRGCv2 \\
\hline $\begin{array}{c}\text { Cook, Chandran } \\
\text { and Fooke s [34] }\end{array}$ & $92.3 \%$ & 147 & FRGCv2 \\
\hline $\begin{array}{c}\text { Lei, B en n moun } \\
\text { and E l-S alla m [32] }\end{array}$ & $95.6 \%$ & 3 & FRGCv2 \\
\hline Zhong, Sun and Tan [15] & $95.6 \%$ & 40 & FRGCv2 \\
\hline Spreeumers [13] & $95.9 \%$ & 30 & FRGCv2 \\
\hline
\end{tabular}

\section{Conclusion}

This paper proposes a novel 3D face recognition approach based on face Factorisation. The experimental study is performed on how discrete regions across the face affect the ability of a subject recognition. The increase in recognition performance occurred when segmenting the face in more regions, corresponds with the hypothesis mentioned in the introduction that it is an interesting approach to split the face into local modules instead of examining the face as a whole. The regionbased 3D face recognition approach is assessed on the FRGCv2 data set containing 
4007 3D face scans from 466 unique subjects, representing a variety of facial expressions. The results of the proposed algorithm outperform those reported by many other state of the arts publication results (Table 2) on the same data set. For future work, this study will be extended to explore gender variations and ethnicity, also work on investigating other methods for data clustering and apply them for $3 \mathrm{D}$ face segmentation.

\section{References}

1. D a n t c h e va, A., P. E li a, A. R o s s. What Else Does Your Biometric Data Reveal? A Survey on Soft Biometrics. - IEEE Transactions on Information Forensics and Security, Vol. 11, 2016, No 3, pp. 441-467.

2. N a s s i h, B., M. Ng a di, A. A m in e, A. E l-A t t a r. New Proposed Fusion between DCT for Feature Extraction and NSVC for Face Classification. - Cybernetics and Information Technologies, Vol. 18, 2018, No 2, pp. 89-97.

3. Th o m p s o n, P. Margaret Thatcher: A New Illusion. Perception, 1980.

4. K 1 ing e n be rg, C. P. Morphometric Integration and Modularity in Configurations of LandMarks: Tools for Evaluating a Priori Hypotheses. - Evolution \& Development, Vol. 11, 2009, No 4, pp. 405-421.

5. X u, C., Y. W a n g, T. T a n, et al. Automatic 3d Face Recognition Combining Global Geometric Features with Local Shape Variation Information. - In: Proc. of 6th IEEE International Conference on Automatic Face and Gesture Recognition, 2004. IEEE, 2004, pp. 308-313.

6. B e n e d i kt, L., D. Coske r, P. L. R o s i n, et al. Assessing the Uniqueness and Permanence of Facial Actions for Use in Biometric Applications. - IEEE Transactions on Systems, Man, and Cybernetics - Part A: Systems and Humans, Vol. 40, 2010, No 3, pp. 44- 460.

7. B l a n z, V., T. V e t t e r. A Morphable Model for the Synthesis of 3d Faces. - In: Proc. of 26th Annual Conference on Computer Graphics and Interactive Techniques, ACM Press/AddisonWesley Publishing Co., 1999, pp. 187-194.

8. He s e 1 t i n e, T., N. P e a r s, J. A u s t i n. Three-Dimensional Face Recognition: An Eigen Surface Approach. - In: 2004 International Conference on Image Processing ICIP'04, 2004, Vol. 2, IEEE, 2004, pp. 1421-1424.

9. A l-O s a i mi, F., M. B e n n a m o u n, A. M i a n. An Expression Deformation Approach to NonRigid 3d Face Recognition. - International Journal of Computer Vision, Vol. 81, 2009, No 3, pp. 302-316.

10. K a k a d i a r i s, I.A., G. P a s s a l i s, G. T o d e r i c i, et al. Three-Dimensional Face Recognition in the Presence of Facial Expressions: An Annotated Deformable Model Approach. IEEE Transactions on Pattern Analysis and Machine Intelligence, Vol. 29, 2007, No 4, pp. 640-649.

11. Z h o u, S., S. X i a o. 3d Face Recognition: A Survey. - Human-Centric Computing and Information Sciences, Vol. 8, 2018, No 1, 35.

12. A mor, B. B., M. Ardabilia n, L. Chen. Enhancing 3d Face Recognition by Mimic's Segmentation. - In: Proc. of 6th International Conference on Intelligent Systems Design and Applications, Vol. 3, IEEE, 2006, pp. 150-155.

13. S p r e e u w e r s, L. Fast and Accurate 3d Face Recognition. - International Journal of Computer Vision, Vol. 93, 2011, No 3, pp. 389-414.

14. Ch a n g, K. I., K. W. B o w y e r, P. J. F1 y n n. Multiple Nose Region Matching for 3D Face Recognition under Varying Facial Expression. - IEEE Transactions on Pattern Analysis and Machine Intelligence, Vol. 28, 2006, No 10, pp. 1695-1700.

15. Z h o n g, C., Z. S u n, T. T a n. Robust 3d Face Recognition Using Learned Visual Codebook. - In: 2007 IEEE Conference on Computer Vision and Pattern Recognition, IEEE, 2007, pp. 1-6.

16. Xi e, Y. L., P. K. Hopke, P. P a at e ro. Positive Matrix Factorisation Applied to a Curve Resolution Problem. - Journal of Chemometrics: A Journal of the Chemometrics Society, Vol. 12, 1998, No 6, pp. 357-364. 
17. Li, X., B. She n, B. D. Li u, et al. Ranking-Preserving Low-Rank Factorisation for Image Annotation with Missing Labels. - IEEE Transactions on Multimedia, Vol. 20, 2018, No 5, pp. 1169-1178.

18. W a n g, Y., X. Li n, L. W u, et al. Robust Subspace Clustering for Multi-View Data by Exploiting Correlation Consensus. - IEEE Transactions on Image Processing, Vol. 24, 2015, No 11, pp. 3939-3949.

19. S a m k o, O., P. L. R o si n, A. D. M a r sh a 11. Robust Automatic Data Decomposition Using a Modified Sparse NMF. - In: International Conference on Computer Vision/Computer Graphics Collaboration Techniques and Applications, Springer, 2007, pp. 225-234.

20. Philli p s, P. J., P. J. Flyn n, T. S c rugg s, et al. Overview of the Face Recognition Grand Challenge. - In: IEEE Computer Society Conference on Computer Vision and Pattern Recognition CVPR'05, 2005, Vol. 1, IEEE, 2005, pp. 947-954.

21. S z e p tycki, P., M. Ardabili a n, L. Chen. A Coarse-to-_ne Curvature Analysis-Based Rotation Invariant 3d Face Landmarking. - In: 3rd International IEEE Conference on Biometrics: Theory, Applications, and Systems, 2009, IEEE, pp. 1-6.

22. Hutto n, T. J., B. F. B u x t o n, P. H a m mond, et al. Estimating Average Growth Trajectories in Shape-Space Using Kernel Smoothing. - IEEE Transactions on Medical Imaging, Vol. 22, 2003, No 6, pp. 747-753.

23. G o w e r, J. C. Generalized Procrustes Analysis. - Psychometrika, Vol. 40, 1975, No 1, pp. 33-51.

24. B o o k s t e i n, F. L. Shape and the Information in Medical Images: A Decade of the Morphometric Synthesis. - Computer Vision and Image Understanding, Vol. 66, 1997, No 2, pp. 97-118.

25. Dudani, S. A. The Distance-Weighted k-Nearest-Neighbor Rule. - IEEE Transactions on Systems, Man, and Cybernetics, 1976, No 4, pp. 325-327.

26. D i n g, C., X. H e, H. D. S i m o n. On the Equivalence of Nonnegative Matrix Factorisation and Spectral Clustering. - In: Proc. of 2005 SIAM International Conference on Data Mining, SIAM, 2005, pp. 606-610.

27. L e e, D. D., H. S. S e u n g. Learning the Parts of Objects by Non-Negative Matrix Factorisation.Nature, Vol. 401, 1999, No 6755, p. 788.

28. S h e n, B., L. S i. Non-Negative Matrix Factorisation Clustering on Multiple Manifolds. - In: AAAI, 2010, pp. 575-580.

29. T u r k, M. A., A. P. P e n t 1 a n d. Face Recognition Using Eigenfaces. - In: Proc. of 1991, IEEE Computer Society Conference on Computer Vision and Pattern Recognition, IEEE, 1991, pp. 586-591.

30. K o t s i a n t i s, S. B., I. Z a h a r a k i s, P. P in t e 1 a s. Supervised Machine Learning: A Review of Classification Techniques. - Emerging Artificial Intelligence Applications in Computer Engineering, Vol. 160, 2007, pp. 3-24.

31. A r lot, S., A. C e li s s e, et al. A Survey of Cross-Validation Procedures for Model Selection. Statistics Surveys, Vol. 4, 2010, pp. 40-79.

32. Le i, Y., M. B e n n m o u n, A. A. E 1-S a 11 a m. An Efficient 3D Face Recognition Approach Based on the Fusion of Novel Local Low-Level Features. - Pattern Recognition, Vol. 46, 2013, No 1, pp. 24-37.

33. F a 1 t e m i e r, T. C., K. W. B o w y e r, P. J. F 1 y n n. A Region Ensemble for 3D Face Recognition. - IEEE Transactions on Information Forensics and Security, Vol. 3, 2008, No 1, pp. 62-73.

34. C o o k, J. A., V. C h a n d r a n, C. B. F o o k e s. 3D Face Recognition Using Log-Gabor Templates. 2006.

Received: 10.03.2019; Second Version: 04.04.2019; Accepted: 19.04.2019 (fast track) 